\title{
Patients' beliefs about adherence to oral antidiabetic treatment: a qualitative study
}

\author{
This article was published in the following Dove Press journal: \\ Patient Preference and Adherence \\ 10 March 2015 \\ Number of times this article has been viewed
}

\author{
Line Guénette ${ }^{1-3}$ \\ Sophie Lauzier ${ }^{1-3}$ \\ Laurence Guillaumie $2-4$ \\ Gabriel Giguère' \\ Jean-Pierre Grégoire ${ }^{1-3}$ \\ Jocelyne Moisan ${ }^{1-3}$ \\ 'Faculty of Pharmacy, Laval University, \\ Quebec City, QC, Canada; ${ }^{2}$ Chair \\ on Adherence to Treatments, Laval \\ University, Quebec City, QC, Canada; \\ ${ }^{3} \mathrm{CHU}$ de Québec Research Center, \\ Population Health and Optimal \\ Practices Research Unit, Quebec City, \\ QC, Canada; ${ }^{4}$ Faculty of Nursing, Laval \\ University, Quebec City, QC, Canada
}

Correspondence: Line Guénette $\mathrm{CHU}$ de Québec Research Center, Population Health and Optimal Health Practices Research Unit, Hôpital du SaintSacrement, 1050 Chemin Sainte-Foy, Quebec City, QC GIS4L8, Canada $\mathrm{Tel}+\mathrm{I} 4 \mid 868275 \mathrm{II}$ extension 4694 Fax +14186827962

Email line.guenette@pha.ulaval.ca
Purpose: The purpose of this study was to elicit patients' beliefs about taking their oral antidiabetic drugs (OADs) as prescribed to inform the development of sound adherenceenhancing interventions.

Methods: A qualitative study was performed. Adults with type 2 diabetes who had been taking an OAD for $>3$ months were solicited to participate in one of six focus groups. Discussions were facilitated using a structured guide designed to gather beliefs related to important constructs of the theory of planned behavior. Four coders using this theory as the theoretical framework analyzed the videotaped discussions.

Results: Forty-five adults participated. The most frequently mentioned advantages for OADtaking as prescribed were to avoid long-term complications and to control glycemia. Family members were perceived as positively influential. Carrying the OAD at all times, having the $\mathrm{OAD}$ in sight, and having a routine were important facilitating factors. Being away from home, not accepting the disease, and not having confidence in the physician's prescription were major barriers to OAD-taking.

Conclusion: This study elicited several beliefs regarding OAD-taking behavior. Awareness of these beliefs may help clinicians adjust their interventions in view of their patients' beliefs. Moreover, this knowledge is crucial to the planning, development, and evaluation of interventions that aim to improve medication adherence.

Keywords: type 2 diabetes, medication adherence, theory of planned behavior, focus groups

\section{Introduction}

Poor metabolic control is often observed in people with type 2 diabetes (T2D). ${ }^{1}$ This occurs despite the availability of several effective and relatively well-tolerated drugs to control this disease and to decrease related comorbidities ${ }^{2}$ and despite an increase in the quality of diabetes care over the years. A frequent barrier to the effective treatment of T2D and other chronic conditions is the suboptimal use of available treatments by patients. In particular, poor adherence to drug treatment is frequent among adults requiring chronic therapy, ${ }^{3}$ including adults using oral antidiabetic drugs (OADs). ${ }^{4}$ In a study of publicly insured people with T2D in Quebec, Canada, we observed that $38 \%$ were nonadherent within their 1 st year of antidiabetic treatment. ${ }^{5}$ Poor adherence represents a major barrier to the metabolic control of T2D, ${ }^{6}$ leads to increased diabetes complications and hospitalizations, ${ }^{2,7}$ and is likely associated with an increase in health care costs. ${ }^{8,9}$

Interventions designed to improve medication adherence in the field of diabetes are therefore needed. It has been suggested that interventions based on psychosocial theories of behavior are more likely to increase the adoption of health-related behaviors. ${ }^{10}$ Among these theories, prediction theories are used to identify the 
variables - determinants - that predict the behavior and should be targeted by interventions. Two meta-analyses revealed that the theory of planned behavior (TPB ${ }^{11}$ is one of the most effective psychosocial theories for predicting the adoption of a behavior. ${ }^{12}$ In the TPB, three important constructs influence the intention of a person to adopt a given behavior: attitude, subjective norm, and perceived behavioral control. Each of these constructs is influenced by a set of beliefs. In the context of adherence to OADs, 1) behavioral beliefs are composed of the perceived advantages and disadvantages of taking OADs as prescribed; 2) normative beliefs are related to the perceived expectation of significant others regarding taking OADs as prescribed; and 3) control beliefs refer to the perceived factors that can impede or facilitate taking OADs as prescribed. Although TPB has been used to predict a wide variety of behaviors, including adherence to medication, ${ }^{13-15}$ studies have rarely used this model in relation to OAD adherence. To our knowledge, only Farmer et al used this model to assess beliefs about hypoglycemic medications in people with T2D in the United Kingdom. ${ }^{16}$ In preparation for the development of adherence-enhancing interventions to be delivered to French-speaking Quebecers, we used a similar approach to identify beliefs about OADtaking in this specific population since these beliefs may vary across different populations. ${ }^{11}$ Our study aimed to elicit the behavioral, normative, and control beliefs of patients regarding taking their OAD as prescribed over 1 month among patients with T2D who initiated these drugs more than 3 months before.

\section{Patients and methods Study design and population}

We performed a qualitative study guided by the TPB to elicit patients' beliefs about OAD-taking. ${ }^{11}$ This study was conducted to subsequently develop a questionnaire to be administered in a quantitative study that aims to identify psychosocial and other predictors of adherence to OADs. The development of such a questionnaire based on the TPB involves a qualitative step in which individuals from the targeted population are questioned about their beliefs regarding the behavior of interest. First, the salient beliefs are identified. The salient beliefs are beliefs that are readily available in memory, which are activated spontaneously without significant cognitive effort when the behavior under study is evocated. ${ }^{17}$ These beliefs are personal, ie, they are expressed by each individual. Then, the modal beliefs are selected among the salient beliefs and used to develop the questionnaire. The modal beliefs are the most commonly held salient beliefs in a given population.

To be eligible, the subjects were required to be 18 years old or older, diagnosed with T2D, and French-speaking. They must have had a prescription for an OAD for more than 3 months before participation and have never used insulin. All OADs were considered, ie, metformin, sulfonylureas, meglitinides, alpha-glucosidase inhibitors, dipeptidyl peptidase-4 (DPP-4) inhibitors, and thiazolidinediones. People with type 1 diabetes or gestational diabetes were excluded. Participants were solicited among students and employees of Laval University, Quebec City, QC, Canada and their relatives (by email invitation) and among members of a local Quebec City diabetic association (by letter invitation). To ensure a certain diversity regarding sociodemographic characteristics among the groups of patients with T2D, letters were sent to members according to their age, sex, and duration of disease. The invitation letter was sent via the association and cosigned by the principal investigator, the study coordinator, and the diabetic association director. Interested individuals were invited to contact the study coordinator by sending a reply card or by telephone. Using a telephone interview guide, the study coordinator then explained the study in more detail and verified study eligibility. Eligible participants were then invited to participate in one of six focus groups. Groups were formed in order to include individuals of both sexes and of different ages and durations of disease. Participants received CAN\$50 for their participation (to cover costs related to parking, transportation, and their time).

Each participant signed a consent form prior to data collection. The Ethics in Research Committee of the CHU de Québec Research Center approved the study.

\section{Data collection and variables}

Six focus groups were held at the offices of the local diabetic association in March 2012. Initially, the participants completed a brief questionnaire containing questions regarding their diabetes (time since diagnosis and treatment), sociodemographic characteristics, and a measure of adherence to OADs. We used a modified version of the validated French version $^{18}$ of the 4-item Morisky Medication Adherence Scale (MMAS-4). ${ }^{19}$ The main modification was in adapting the questionnaire to OADs as suggested by the developers.

Discussions were facilitated by a member of our team (Laurence Guillaumie) assisted by another team member (GG) using a structured guide designed to gather information on behavioral, normative and control beliefs explored in the 
study. The target behavior for the discussions was to take all OADs exactly as prescribed every day. All questions of the structured guide were formulated according to the recommendations of the TPB developers ${ }^{11}$ and referred to this targeted behavior. Behavioral beliefs were obtained with the following question: "In the next month, what would be for you the advantages/disadvantages of taking all of your OADs exactly as prescribed every day?" Normative beliefs were identified by asking the following question: "In your opinion, which person or group of persons would approve/disapprove of your taking all of your OADs exactly as prescribed every day over the next month?" Control beliefs that include barriers and facilitating factors were elicited with "What would impede/ facilitate your taking of all your OADs exactly as prescribed every day in the next month?" Each focus group was videotaped. The director of the association was present to answer at the end of the sessions any questions raised about diabetes and treatment. The director's presence was also necessary to ensure that potential erroneous information about diabetes and OADs that would be shared during the sessions and that may negatively influence participants in their future medicationtaking behavior could be corrected. To avoid influencing the discussions, these interventions were only done after the end of the focus groups.

\section{Analysis}

To identify modal beliefs, we used the procedure recommended by the TPB developers. ${ }^{11} \mathrm{We}$ conducted a content analysis of the discussions using the TPB as the theoretical framework. First, three authors (Laurence Guillaumie, SL, GG) individually viewed all of the tapes and transcribed integrally every mention of a specific belief; this procedure was followed for each category of beliefs (ie, behavioral, normative, and control beliefs). Second, similar beliefs in each category were grouped to form mutually exclusive beliefs. A frequency of mention was attributed to each of these mutually exclusive beliefs. For each category of beliefs, modal beliefs were defined as those mutually exclusive beliefs with the highest frequency of mention (in \%) until the total frequency reached $75 \%$. The three authors met during this process to reach agreement on the final modal beliefs identified. Moreover, Lilianne Bordeleau listened to all audiotapes to extract relevant quotations illustrating the modal beliefs in more detail. The focus groups were conducted in French, and a professional translator translated the quotations presented in this article.

\section{Results}

A total of 45 subjects with T2D participated in the six focus groups, with a mean of eight participants per group (range: 6-9). Each session lasted approximately 2 hours. Of these participants, 26 (57.8\%) were men, and the mean age was 63.8 years (range: 39-78). Participants had had diabetes for a mean of 8.2 years (range: $2-22$ ) and $89 \%$ reported using metformin alone or in combination with another OAD. Based on the MMAS-4, 18 participants (40\%) were considered adherent (score $=4 / 4$ ) and the mean score was 3.16/4. The participant characteristics per focus group are presented in Table 1.

The groups allowed us to identify many salient beliefs. In this section, modal beliefs (ie, those salient beliefs that were the most commonly held) are presented. Seven behavioral beliefs were identified as modal and are presented in Table 2, along with quotations. All beliefs identified were advantages, except for experiencing side effects (mainly gastrointestinal problems and hypoglycemia), which was the only disadvantage mentioned. The main advantage was to avoid long-term complications of diabetes, such as amputations and blindness.

Participants identified three groups as particularly important for their OAD adherence (ie, modal normative beliefs): spouses, children, and family in the broad sense (Table 3). All of them approved of their OAD-taking as prescribed. Health care professionals and support groups were not identified as the main influences for this behavior, although some respondents mentioned physicians and

Table I Participant characteristics according to the focus groups $(n=45)$

\begin{tabular}{|c|c|c|c|c|c|c|c|c|c|}
\hline \multirow{2}{*}{$\begin{array}{l}\text { Focus } \\
\text { group } \\
\text { number }\end{array}$} & \multirow[t]{2}{*}{$\begin{array}{l}\text { Number of } \\
\text { participants }\end{array}$} & \multicolumn{2}{|l|}{ Sex } & \multicolumn{2}{|c|}{$\begin{array}{l}\text { Age } \\
\text { (years) }\end{array}$} & \multicolumn{2}{|c|}{$\begin{array}{l}\text { Years with } \\
\text { type } 2 \text { diabetes }\end{array}$} & \multicolumn{2}{|c|}{$\begin{array}{l}\text { Adherent according } \\
\text { to MMAS-4 }\end{array}$} \\
\hline & & Men & Women & $<65$ & $\geq 65$ & $\leq \mathbf{5}$ & $>5$ & Yes & No \\
\hline I & 9 & 6 & 3 & 5 & 4 & 4 & 5 & 2 & 7 \\
\hline 2 & 7 & 5 & 2 & 3 & 4 & 3 & 4 & 3 & 4 \\
\hline 3 & 6 & 3 & 3 & 3 & 3 & 0 & 6 & 5 & I \\
\hline 4 & 8 & 5 & 3 & 4 & 4 & 4 & 4 & 3 & 5 \\
\hline 5 & 7 & 4 & 3 & 3 & 4 & 2 & 5 & 3 & 4 \\
\hline 6 & 8 & 3 & 5 & 3 & 5 & 3 & 5 & 2 & 6 \\
\hline
\end{tabular}

Abbreviation: MMAS-4, 4-item Morisky Medication Adherence Scale. 
Table 2 Modal behavioral beliefs of the 45 type 2 diabetes patients who participated in the focus groups, in decreasing order of frequency

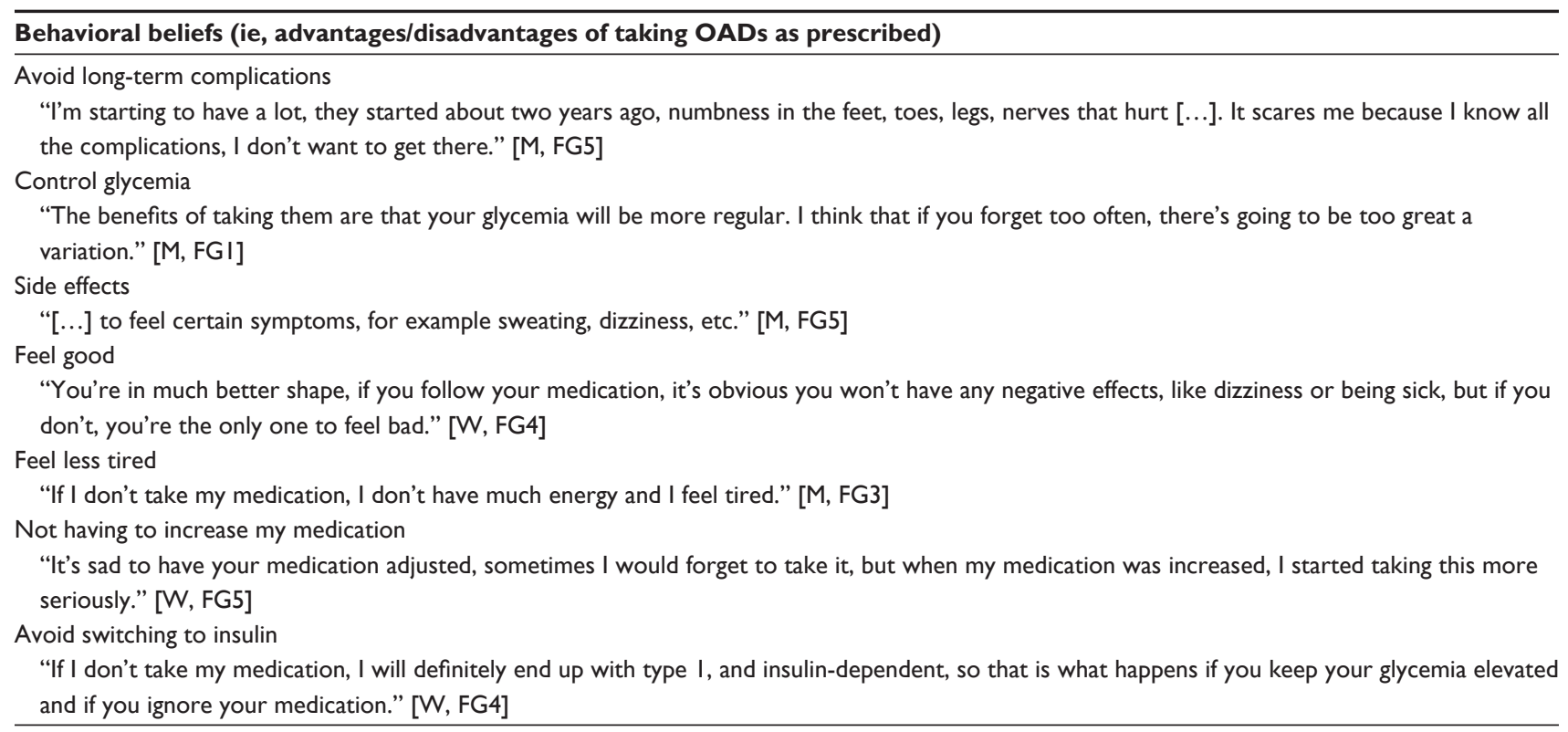

Note: Participant codes definition: M, Man; W, Woman; FG, Focus group, and the number refers to the focus group number.

Abbreviation: OADs, oral antidiabetic drugs.

pharmacists. Some people mentioned that they felt guilty and embarrassed when they had to take their drugs in front of others (eg, in a restaurant) because drug-taking was perceived as socially disapproved. However, this latter belief was not modal in this population.

Ten factors were identified as modal control beliefs constituting six facilitators and four barriers (Table 4). Among the most frequent factors that would aid in taking the treatment as prescribed were: always having the drugs on hand (eg, in a purse, in their pockets, in their car); having the drugs on the kitchen counter in plain sight; and having a routine (ie, associating drug-taking with something one does every day). The main factors that would prevent participants from taking the treatment as prescribed were being away or on vacation or at a restaurant, not accepting their disease, and having no confidence in the physician's prescription (eg, the number of drugs and dosage). Among other barriers (not identified as modal), some participants mentioned that it was quite difficult to see their physician and that when they had the opportunity to see him or her, the consultation was too short to ask questions and address concerns. Others mentioned that they sometimes forgot to take their OADs and that some occasions were more critical than others for forgetfulness (eg, lunchtime or dinner were more difficult compared with the morning). Forgetfulness was also observed in the MMAS-4 results. Among the 27 nonadherent participants, 17 (63\%) identified forgetfulness (the first question in the MMAS-4 questionnaire) as the only reason for being nonadherent.

\section{Discussion}

Our objective was to qualitatively elicit patients' beliefs regarding OAD-taking to inform the elaboration of a questionnaire to assess the psychosocial determinants of $\mathrm{OAD}$ adherence in a quantitative study. First, the participants

Table 3 Modal normative beliefs of the 45 type 2 diabetes patients who participated in the focus groups, in decreasing order of frequency

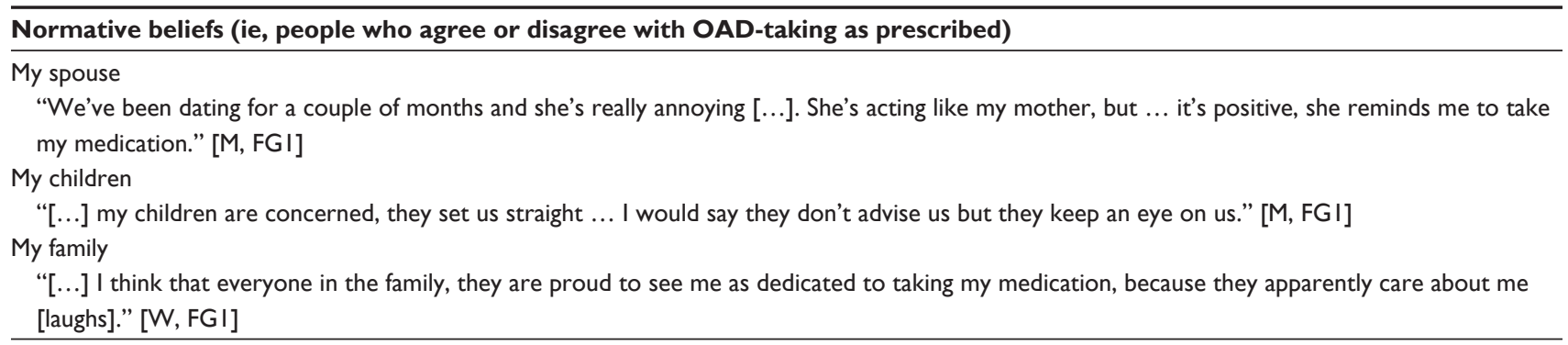

Note: Participant codes definition: M, Man; W, Woman; FG, Focus group, and the number refers to the focus group number.

Abbreviation: OAD, oral antidiabetic drug. 
Table 4 Modal control beliefs of the 45 type 2 diabetes patients who participated in the focus groups, in decreasing order of frequency

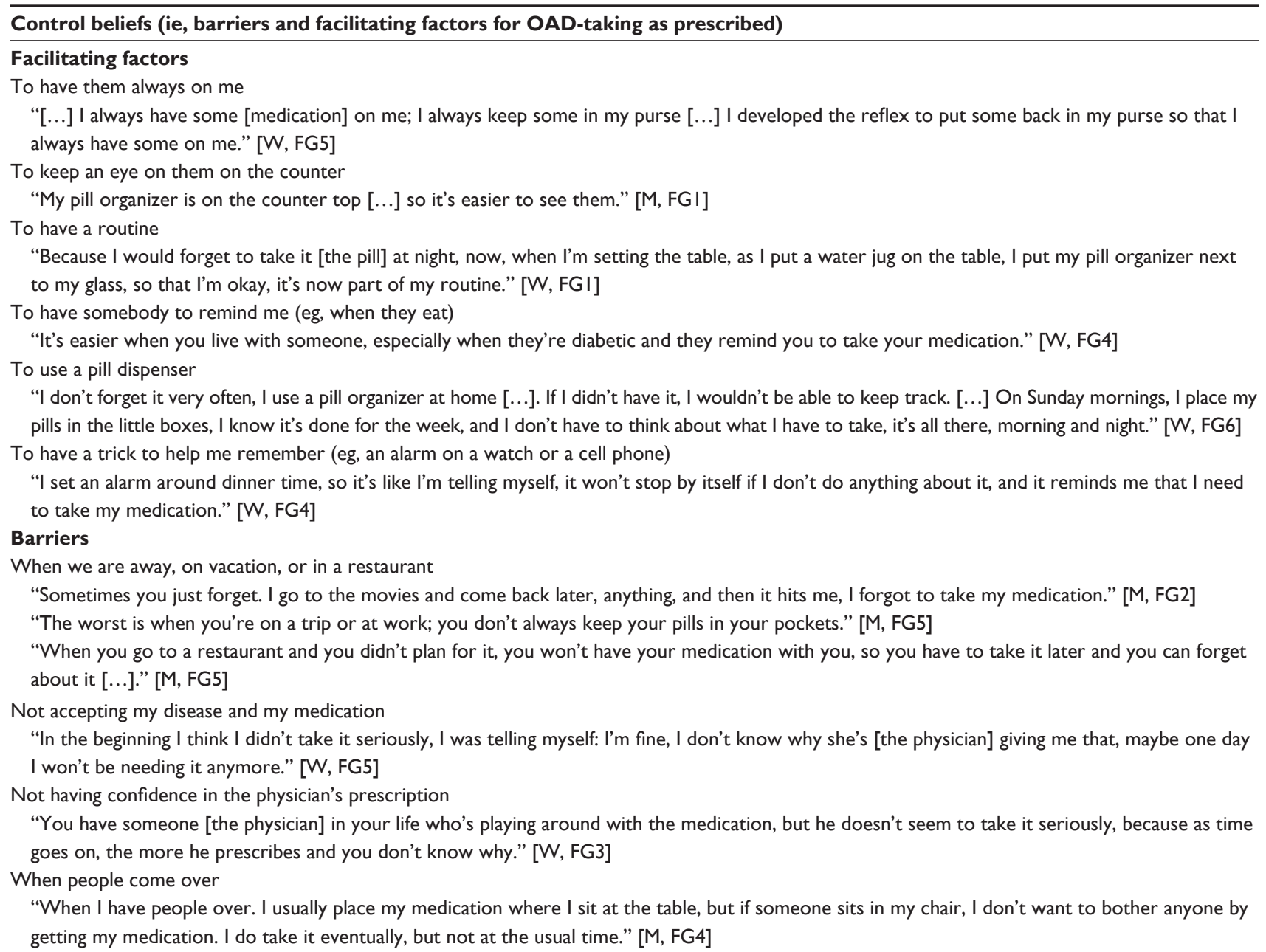

Note: Participant codes definition: M, Man; W, Woman; FG, Focus group, and the number refers to the focus group number.

Abbreviation: OAD, oral antidiabetic drug.

reported that taking OADs as prescribed over the next month would generally have positive consequences, and they described several short- and long-term benefits. Similar results were observed in the only similar study that we identified. ${ }^{16}$ Many participants in both that study and ours mentioned that they were taking their drugs regularly to control their diabetes or their glycemia, suggesting that the understanding and perceived efficacy of these medications may positively influence adherence to OADs.

The only disadvantage of taking the OADs as prescribed was to experience some side effects of these drugs. Gastrointestinal problems, which frequently occur with metformin the first-line agent for $\mathrm{T}^{2} \mathrm{D}^{20}$ - were commonly mentioned by participants and could impede OAD adherence. ${ }^{21}$ This finding suggests that clinicians should inform their patients about this side effect that typically occurs during the first days following metformin treatment initiation, after an increase in the dosage, and when using high doses. Hypoglycemia, which can occur with insulin secretagogues, ${ }^{20}$ was also frequently alluded to by participants. They associated this side effect to certain OADs (that were too potent) or to the dose. The risk of hypoglycemia should be balanced with positive short- and long-term benefits that are significant for OAD adherence because this balance has been reported to be associated with higher rates of adherence..$^{22}$ Considering that the only disadvantage of taking the OADs as prescribed was to experience some side effects, the management of such effects should be clearly presented and systematically assessed to avoid limiting an individual's ability to adhere. In their study, Farmer et al observed that $32.8 \%$ of participants believed that taking their diabetes medications regularly would cause them unpleasant side effects, but this belief was not associated with medication adherence. ${ }^{16}$ In contrast to the observations in that study, ${ }^{16}$ weight gain was not a modal belief held by our population regarding OAD adherence. This difference in results might have occurred because, in 
contrast to these authors, we excluded patients taking insulin, a drug commonly associated with weight gain..$^{20,23}$

Health care professionals should be aware that some of the advantages of taking OADs as prescribed that were noted by the participants were clinically incorrect. Indeed, participants stated that they were following this behavior to avoid an increase in their medication (which would increase the management burden) or to avoid switching to insulin (signifying that they would need to inject a drug). This latter belief was also reported by $86.4 \%$ of people in the Farmer et al study. ${ }^{16}$ Although these beliefs were perceived as beneficial to OAD adherence, health care professionals should make their patients aware that an increase in medication or a switch to insulin is not a failure of the patient's management of his/her diabetes, as perceived by many persons with $\mathrm{T} 2 \mathrm{D},{ }^{24}$ but rather a consequence of the evolution of the disease. This feeling of failure may lead to discouragement and to further nonadherence if one experiences an increase in his/her medication despite being perfectly OAD-adherent. ${ }^{23}$

Regarding normative beliefs, participants identified their spouse and family as the most important person and/or group(s) for their OAD adherence behavior. These people helped them remember to take their medication and were concerned about other behaviors related to their diabetes control. For example, many participants discussed the importance of their spouse in relation to dieting. Social support and family support by spouses and family members have also been associated positively with medication adherence in other disease ${ }^{25}$ and with better glycemic control in patients with T2D. ${ }^{3}$ Health care professionals and support groups were not identified as influential for medication adherence in our study. This suggests that interventions to improve medication OAD adherence should attempt to involve significant relatives.

In addition to having someone to remind them to take their medication, the participants identified other facilitators of their OAD-taking. All of them were related to two themes, ie, having the drugs available (always on me, on the kitchen counter in plain sight) and having cues to remember (a routine, tricks). "Having a regular routine would make it easier for me to take my medication regularly" was also a frequent control belief in the Farmer et al study. ${ }^{16}$ Two of the barriers identified were also associated with routine, specifically those events that disrupted the routine of taking the OAD as prescribed (ie, being away, having someone at home). This was also observed in a study among patients with T2D from community pharmacies in the Netherlands. ${ }^{26}$ The act of taking a drug is a relatively simple behavior in comparison with exercising or dieting. This might suggest that forgetfulness is one of the main reasons for not being adherent to OAD when the motivation or intention is present. This observation was reported in a review of patients with heart failure. ${ }^{27}$ In that regard, interventions to improve adherence should be directed toward helping people integrate their medication-taking into their routine and facilitating drug availability (eg, by facilitating renewals or by educating patients to carry a sample of their drugs in almost all circumstances), particularly when their routine is disrupted. To facilitate routine, cues to remember such as placing the pills in a place where a daily activity is conducted (eg, brushing teeth, preparing meals) might be suggested. To improve drug availability, services such as home delivery or the synchronization of refills could be offered.

However, two barriers were not related to forgetfulness: having no confidence in the physician's prescription and not accepting the disease. These might instead be related to the physician-patient relationship and to the physician's communication skills, as suggested by others. ${ }^{28-30}$ Physician communication style influences patient knowledge and initial beliefs about the medication, follow-up attendance, and treatment satisfaction after attempting medication use. ${ }^{28}$ Communication style and patient satisfaction are both predictive of better medication adherence. ${ }^{28}$ Poor physician-patient relationships and poor communication were also found to be associated with poor adherence $e^{30}$ and poor glucose control. ${ }^{29}$ Physicians should be aware that distrust in their prescriptions occurs more frequently than they think, particularly among well-educated patients, and is not always explicitly expressed. ${ }^{31}$ Adopting a collaborative style and shared decision making to foster a collaborative relationship in which the provider facilitates or enables the client to take an active role in his or her treatment have been suggested as possible solutions..$^{28,31}$

One of the strengths of this study is the utilization of a theoretical framework - the TPB - both in its conceptualization and during the analysis. Indeed, it is one of the first studies to elicit patients' beliefs regarding their OAD adherence based on a conceptual model. Moreover, we had a sufficient number of participants to perform this type of study (according to the TPB developers, 30 participants is sufficient), ${ }^{11,17}$ and the patients had different characteristics that were likely to influence adherence behaviors and the related beliefs. However, our results may not apply to all people with diabetes, as some subgroups were not represented in our study sample, eg, persons newly treated with 
OADs, those with type 1 diabetes, or those being treated with insulin or with non-OADs. Further studies will be needed to elicit beliefs in those populations. Additionally, most of the participants were members of a diabetic association. These individuals are likely to be more motivated and informed than the general population with T2D or to experience more unmet information and support needs regarding their illness and treatment. Moreover, a large proportion of nonadherent participants identified forgetfulness as the main reason for their behavior (ie, non-intentional nonadherence). Consequently, the beliefs gathered from our participants may be different from the beliefs of less-motivated patients or those of intentional nonadherers. Additionally, this study was conducted to develop a questionnaire based on the TPB. In accordance with this objective, a structured interview guided the focus group discussions. Whereas this protocol allowed us to obtain important information on beliefs related to the TPB, we may have overlooked some other types of beliefs influencing OAD-taking. Finally, this was a qualitative study designed to elicit modal beliefs. Quantitative studies are needed to measure their association with OAD adherence.

\section{Conclusion}

This study elicited several beliefs regarding OAD-taking behavior. Awareness of these beliefs may help clinicians who are treating patients taking OADs adjust their interventions in view of their patients' beliefs. Clinicians should particularly emphasize the short- and long-term benefits of taking OADs as prescribed. They should inform their patients with T2D about the management of side effects and the natural course of this disease. Relatives should be involved because they may positively influence OAD adherence. Clinicians should also help people integrate their medication-taking into their routine and facilitate drug availability. Moreover, clinicians should be aware that their communication skills are particularly important in this context. Clinicians should be approachable and supportive; should ask about and listen to their patients' views and concerns; and should use a shared decision-making model of care.

\section{Acknowledgments}

The Laval University Chair on Adherence to Treatments funded this study. This Chair is supported by non-restricted grants from AstraZeneca Canada, Merck Canada, Sanofi Canada, Pfizer Canada, and from the Prends soin de toi program (a Quebec provincial program for the improvement of public health). We wish to thank Lilianne Bordeleau, who extracted the relevant quotations; Alexandre Audet, the professional translator who translated the quotations; American Journal Experts who edited the text; and the local diabetic association Les Diabétiques de Québec, particularly their director Helene House, who made the recruitment for this study possible. We are also grateful to all of the participants.

\section{Disclosure}

The authors report no conflicts of interest in this work. The companies supporting the Chair on Adherence to Treatments had no role in the study design, data collection and analysis, decision to publish, or preparation of the manuscript.

\section{References}

1. Leiter LA, Berard L, Bowering CK, et al. Type 2 diabetes mellitus management in Canada: is it improving? Can J Diabetes. 2013;37(2): 82-89.

2. [No authors listed]. Effect of intensive blood-glucose control with metformin on complications in overweight patients with type 2 diabetes (UKPDS 34). UK Prospective Diabetes Study (UKPDS) Group. Lancet. 1998;352(9131):854-865.

3. DiMatteo MR. Variations in patients' adherence to medical recommendations: a quantitative review of 50 years of research. Med Care. 2004; 42(3):200-209.

4. Cramer JA. A systematic review of adherence with medications for diabetes. Diabetes Care. 2004;27(5):1218-1224.

5. Guénette L, Moisan J, Breton MC, Sirois C, Grégoire JP. Difficulty adhering to antidiabetic treatment: factors associated with persistence and compliance. Diabetes Metab. 2013;39(3):250-257.

6. Pladevall M, Williams LK, Potts LA, Divine G, Xi H, Lafata JE. Clinical outcomes and adherence to medications measured by claims data in patients with diabetes. Diabetes Care. 2004;27(12):2800-2805.

7. Lau DT, Nau DP. Oral antihyperglycemic medication nonadherence and subsequent hospitalization among individuals with type 2 diabetes. Diabetes Care. 2004;27(9):2149-2153.

8. Salas M, Hughes D, Zuluaga A, Vardeva K, Lebmeier M. Costs of medication nonadherence in patients with diabetes mellitus: a systematic review and critical analysis of the literature. Value Health. 2009;12(6): 915-922.

9. Lee WC, Balu S, Cobden D, Joshi AV, Pashos CL. Prevalence and economic consequences of medication adherence in diabetes: a systematic literature review. Manag Care Interface. 2006;19(7):31-41.

10. Glanz K, Bishop DB. The role of behavioral science theory in development and implementation of public health interventions. Annu Rev Public Health. 2010;31:399-418.

11. Ajzen I. The theory of planned behavior. Organ Behav Hum Decis Process. 1991;50:179-211.

12. Armitage CJ, Conner M. Efficacy of the Theory of Planned Behaviour: a meta-analytic review. Br J Soc Psychol. 2001;40(Pt 4):471-499.

13. Chisholm MA, Williamson GM, Lance CE, Mulloy LL. Predicting adherence to immunosuppressant therapy: a prospective analysis of the theory of planned behaviour. Nephrol Dial Transplant. 2007;22(8): 2339-2348.

14. Hugon A, Roustit M, Lehmann A, et al. Influence of intention to adhere, beliefs and satisfaction about medicines on adherence in solid organ transplant recipients. Transplantation. 2014;98(2):222-228.

15. Vissman AT, Hergenrather KC, Rojas G, Langdon SE, Wilkin AM, Rhodes SD. Applying the theory of planned behavior to explore HAART adherence among HIV-positive immigrant Latinos: elicitation interview results. Patient Educ Couns. 2011;85(3):454-460. 
16. Farmer A, Kinmonth AL, Sutton S. Measuring beliefs about taking hypoglycaemic medication among people with type 2 diabetes. Diabet Med. 2006;23:265-270.

17. Godin G. Les Comportements dans le Domaine de la Santé: Comprendre pour Mieux Intervenir. [Behavior in the field of health: understanding for better action]. Montreal: Les Presses de 1'Université de Montréal; 2012. French.

18. Grégoire JP, Guibert R, Archambault A, Contandriopoulos AP. Medication compliance in a family practice: testing a self-report questionnaire in a primary care setting. Can Fam Physician. 1992;38:2333-2337.

19. Morisky DE, Green LW, Levine DM. Concurrent and predictive validity of a self-reported measure of medication adherence. Med Care. 1986; 24(1):67-74.

20. Canadian Diabetes Association Clinical Practice Guidelines Expert Committee. Canadian Diabetes Association 2008 Clinical Practice Guidelines for the Prevention and Management of Diabetes in Canada. Can J Diabetes. 2008;32(Suppl 1):S1-S201.

21. Mann DM, Ponieman D, Leventhal H, Halm EA. Predictors of adherence to diabetes medications: the role of disease and medication beliefs. J Behav Med. 2009;32(3):278-284.

22. Horne R, Weinman J. Patients' beliefs about prescribed medicines and their role in adherence to treatment in chronic physical illness. J Psychosom Res. 1999;47(6):555-567.

23. Ross SA. Breaking down patient and physician barriers to optimize glycemic control in type 2 diabetes. Am J Med. 2013;126(9 Suppl 1): S38-S48.

24. Grant RW, Pabon-Nau L, Ross KM, Youatt EJ, Pandiscio JC, Park ER. Diabetes oral medication initiation and intensification: patient views compared with current treatment guidelines. Diabetes Educ. 2011;37(1): 78-84.
25. Kardas P, Lewek P, Matyjaszczyk M. Determinants of patient adherence: a review of systematic reviews. Front Pharmacol. 2013;4:91.

26. Vervloet M, Spreeuwenberg P, Bouvy ML, Heerdink ER, de Bakker DH, van Dijk L. Lazy Sunday afternoons: the negative impact of interruptions in patients' daily routine on adherence to oral antidiabetic medication. A multilevel analysis of electronic monitoring data. Eur J Clin Pharmacol. 2013;69(8):1599-1606.

27. Wu JR, Moser DK, Lennie TA, Burkhart PV. Medication adherence in patients who have heart failure: a review of the literature. Nurs Clin North Am. 2008;43(1):133-153; vii-viii

28. Bultman DC, Svarstad BL. Effects of physician communication style on client medication beliefs and adherence with antidepressant treatment. Patient Educ Couns. 2000;40(2):173-185.

29. Ciechanowski PS, Katon WJ, Russo JE, Walker EA. The patientprovider relationship: attachment theory and adherence to treatment in diabetes. Am J Psychiatry. 2001;158(1):29-35.

30. Ratanawongsa N, Karter AJ, Parker MM, et al. Communication and medication refill adherence: the Diabetes Study of Northern California. JAMA Intern Med. 2013;173(3):210-218.

31. Bezreh T, Laws MB, Taubin T, Rifkin DE, Wilson IB. Challenges to physician-patient communication about medication use: a window into the skeptical patient's world. Patient Prefer Adherence. 2012;6: $11-18$.
Patient Preference and Adherence

\section{Publish your work in this journal}

Patient Preference and Adherence is an international, peer-reviewed, open access journal that focuses on the growing importance of patient preference and adherence throughout the therapeutic continuum. Patient satisfaction, acceptability, quality of life, compliance, persistence and their role in developing new therapeutic modalities and compounds to optimize

\section{Dovepress}

clinical outcomes for existing disease states are major areas of interest for the journal. This journal has been accepted for indexing on PubMed Central. The manuscript management system is completely online and includes a very quick and fair peer-review system, which is all easy to use. Visit http://www. dovepress.com/testimonials.php to read real quotes from published authors. 